\title{
Connective Eccentric Index of an infinite family of linear polycene parallelogram benzenoid
}

\author{
Mohammad Reza Farahani \\ Department of Applied Mathematics, Iran University of Science and Technology (IUST), \\ Narmak, Tehran 16844, Iran \\ E-mail address:Mr_Farahani@Mathdep.iust.ac.ir,MrFarahani88@Gmail.com
}

\begin{abstract}
Let $G=(V, E)$ be a graph, where $V(G)$ is a non-empty set of vertices and $E(G)$ is a set of edges. We defined $d_{v}$ denote the degree of vertex $v \in V(G)$. The Eccentric Connectivity index $\xi(G)$ and the Connective Eccentric index $C^{\xi}(G)$ of graph $G$ are defined as $\xi(G)=\sum_{v \in V(G)} d_{v} \times \varepsilon(v)$ and $C^{\xi}(G)=$ $\sum_{v \in V(G)} d_{v} \times \varepsilon(v)^{-1}$, where $\varepsilon(v)$ is defined as the length of a maximal path connecting a vertex $v$ to another vertex of $G$. In this present paper, we compute these Eccentric indices for an infinite family of linear polycene parallelogram benzenod by a new method.
\end{abstract}

Keywords: Molecular graphs; Linear polycene parallelogram; Benzenoid; Eccentric connectivity index; Connective eccentric index

\section{INTRODUCTION}

Let $G$ be a molecular graph, the vertex and edge sets of a graph $G$ are denoted by $V(G)$ and $E(G)$, respectively. A topological index is a numeric quantity related to $G$ which is invariant under graph automorphisms. In theoretical chemistry molecular structure descriptor or topological indices, are used to compute properties of chemical compounds.

The Wiener index is the first topological index proposed to be used in chemistry. It was introduced in 1947 by Chemist Harold Wiener, as the path number for characterization of alkanes. It is defined as the sum of distances between all pairs of vertices in the graph under consideration [1-3]. If $x, y \in V(G)$ then the distance $d(x, y)$ between $x$ and $y$ is defined as the length of a minimum path connecting $x$ and $y$. Then the Wiener index is equale to

$$
W(G)=\frac{1}{2} \sum_{u \in V(G)} \sum_{v \in V(G)} d(u, v)
$$


The Eccentric Connectivity index $\xi(G)$ of the molecular graph $G$, was proposed by Sharma, Goswami and Madan [4]. It is defined as

$$
\xi(G)=\sum_{v \in V(G)} d_{v} \times \varepsilon(v)
$$

where $d_{v}$ denotes the degree of the vertex $v$ in $G$ and $\varepsilon(v)=\operatorname{Max}\{d(v, u) \mid \forall v \in V(G)\}$ denote the largest distance between $v$ and any other vertex $u$ of $G$ [5-14]. The radius and diameter of $G$ are defined as the minimum and maximum eccentricity among vertices of $G$, respectively. In other words,

$$
\begin{gathered}
D(G)=\operatorname{Max}_{v \in V(G)}\{d(u, v) \mid \forall u \in V(G)\} \\
R(G)=\operatorname{Min}_{v \in V(G)}\{\operatorname{Max}\{d(u, v) \mid \forall u \in V(G)\}\}
\end{gathered}
$$

The Eccentric Connectivity polynomial of a graph $G$, was defined by Alaeiyan, Mojarad and Asadpour as follows [15]:

$$
\operatorname{ECP}(G, x)=\sum_{v \in V(G)} d_{v} x^{e c c(v)}
$$

Then the eccentric connectivity index is the first derivative of $\operatorname{ECP}(G, x)$ evaluated at $x=1$. as follows:

The Connective Eccentric index $C^{\xi}(G)$ was defined by Gupta, Singh and Madan [16,17]

$$
C^{\xi}(G)=\sum_{v \in V(G)} \frac{d_{v}}{\varepsilon(v)}
$$

where $d_{v}, \varepsilon(v)$ denote the degree and eccentric of vertex $v$ in $G$.

\section{RESULTS AND DISCUSSION}

In this section is to compute the Eccentric Connectivity $\xi(G)$ and Connective Eccentric $C^{\xi}(G)$ indices, for an infinite family of linear polycene parallalogram of benzenoid graph [18], by continue the results from [15,19-24]. This benzenoid graph has $2 n(n+2)$ vertices and $3 n 2+4 n-1$ edges. The general representation of linear polycene parallalogram of benzenoid $P(n, n)$ is shown in Figure 1. Thus, we have following theorem:

Theorem 1. Let $G$ be the linear polycene parallalogram of benzenoid $P(n, n)(\forall n \in \mathbb{N})$. Then, the Eccentric Connectivity index $\xi(G)$ of $P(n, n)$ is equal to

$$
\xi(P(n, n))=16 n^{3}+15 n^{2}-75 n+6
$$

The Connective Eccentric index $C^{\xi}(G)$ of $P(n, n)$ is equal to 


$$
C^{\xi}(P(n, n))=\sum_{i=0}^{n-2}\left(\frac{5 i^{2}+(16 n+1) i+(11 n+7) n}{2 i^{3}+3(2 n+1) i^{2}+\left(6 n^{2}+6 n+1\right) i+\left(2 n^{2}+3 n+1\right) n}\right)+\left(\frac{16 n^{2}-10 n+3}{n\left(8 n^{2}+2 n-1\right)}\right)
$$

Proof: By considering the general form of linear polycene parallalogram benzenoid graph $G=P(n, n) \quad(\forall n \geq 1)$ depicted in Figure 1 and refer to $[15,19]$, we have the maximum eccentric connectivity and minimum eccentric connectivity for a $v \in V(P(n, n))$ as $\operatorname{Max}_{\varepsilon(v)}=4 n-1$ and $\operatorname{Min}_{\varepsilon(v)}=2 n$.

Also, from Figure 1 and Table 1, one can see that all vertices as degree two $\left(d_{v}=2\right)$ have eccentric connectivity index $4 n-1,4 n-2,4 n-4,4 n-6, \ldots, 2 n+2,2 n+1$ and for all other vertices as degree three $\left(d_{v}=3\right)$ have other eccentric connectivity index between $4 n-3$ until $2 n$.

Now, by according to Figure 1 and using the results in Table 1, we have following computations:

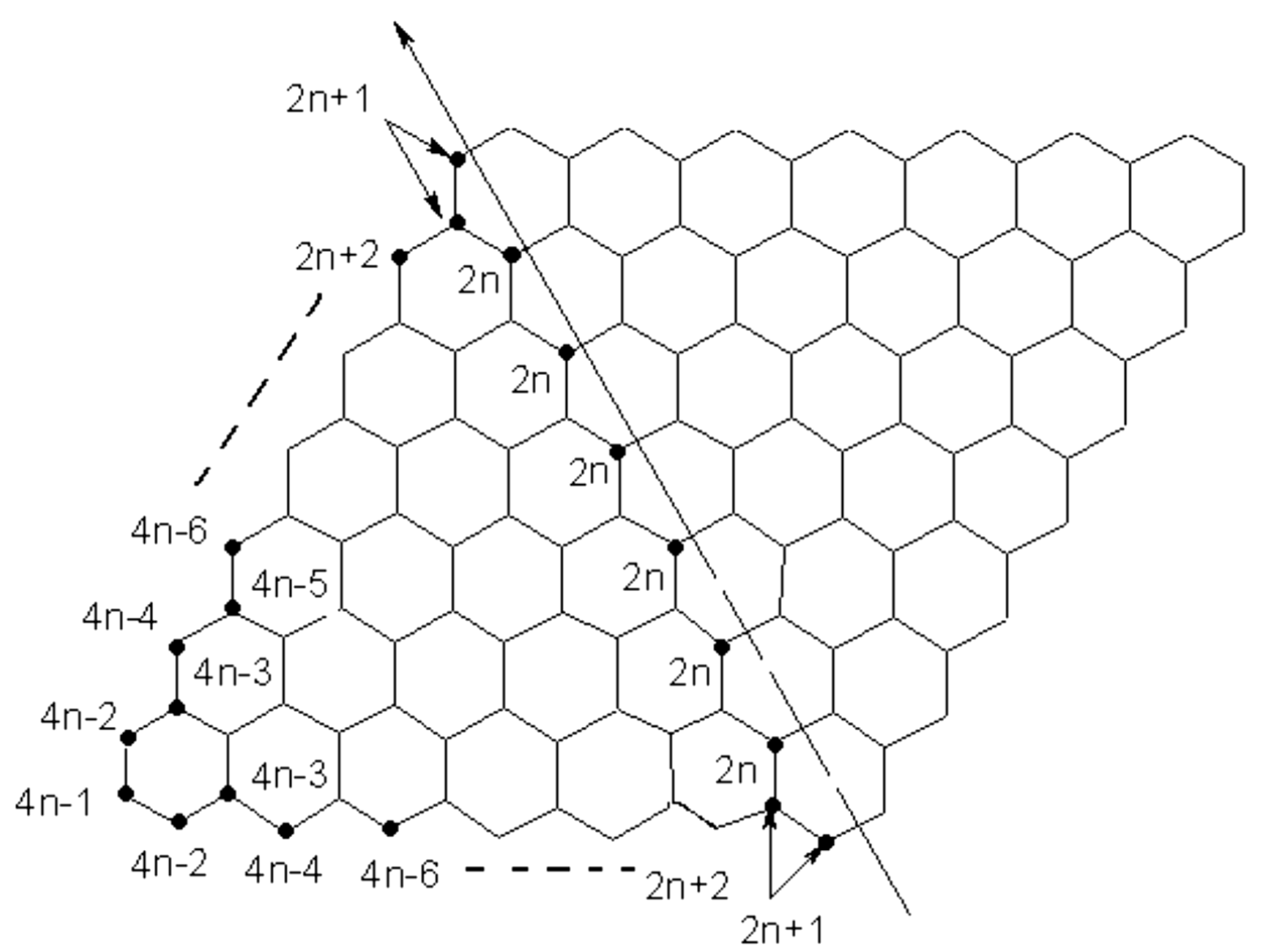

Figure 1. The general representation of linear polycene parallalogram of benzenoid $P(n, n)$ and the eccentric connectivity of its vertices [19]. 
Table 1. Eccentric connectivity index for all vertices of linear polycene parallalogram benzenoid graph $G=P(n, n)$.

\begin{tabular}{|c|c|c|c|c|c|c|c|c|}
\hline $2 n+1$ & $2 n+1$ & $2 n+2$ & $\ldots$ & $4 n-5$ & $4 n-4$ & $4 n-3$ & $4 n-2$ & $4 n-1$ \\
\hline $2 n$ & $2 n+1$ & $2 n+2$ & $\ldots$ & $4 n-5$ & $4 n-4$ & $4 n-3$ & $4 n-2$ & \\
\hline $2 n$ & $2 n+1$ & $2 n+2$ & $\ldots$ & $4 n-5$ & $4 n-4$ & & & \\
\hline $2 n$ & $2 n+1$ & $2 n+2$ & $\ldots$ & & & & & \\
\hline$\ldots$ & $\ldots$ & $\ldots$ & & & & & & \\
\hline$\ldots$ & $\ldots$ & $\ldots$ & & & & & & \\
\hline$\ldots$ & $\ldots$ & $\ldots$ & & & & & & \\
\hline $2 n$ & $2 n+1$ & $2 n+2$ & & & & & & \\
\hline & $2 n+1$ & & & & & & & \\
\hline
\end{tabular}

$$
\begin{aligned}
\xi(P(n, n)) & =\sum_{v \in V(P(n, n))} d_{v} \times \varepsilon(v) \\
& =2 \times[2(4 n-1)+4 \times(4 n-2)+4 \times(4 n-4)+4 \times(4 n-6)+\ldots+4 \times(2 n+4)+4 \times(2 n+2) \\
& +4 \times(2 n+1)]+3 \times[2(n-1) \times(2 n)+2 n \times(2 n+1)+2(n-1) \times(2 n+2)+2(n-1) \times(2 n+3) \\
& +2(n-2) \times(2 n+4)+2(n-2) \times(2 n+5)+2(n-3) \times(2 n+6)+2(n-3) \times(2 n+7)+\ldots \\
& +6 \times(4 n-6)+6 \times(4 n-5)+4 \times(4 n-4)+4 \times(4 n-3)] \\
& =4(4 n-1)+8 \times(4 n-2)+8 \times(4 n-4)+8 \times(4 n-6)+\ldots+8 \times(2 n+4)+8 \times(2 n+2) \\
& +8 \times(2 n+1)+6(n)[2 n+2 n+1]+6(n-1)[2 n+2+2 n+3]+6(n-2)[2 n+4+2 n+5] \\
& +\ldots+18[4 n-6+4 n-5]+12[4 n-4+4 n-3]-12 n \\
& =8 \sum_{i=1}^{n-2}(2 i+2 n+2)+32 n+4+6 \sum_{i=0}^{n-2}\left(4 n^{2}+n-4 i^{2}-i\right)-12 n \\
& =8\left(3 n^{2}-5 n-2\right)+32 n+4-6\left[(n-2)\left(\frac{8 n^{2}-17 n+9}{6}\right)-n(n-2)(4 n+1)\right]-12 n \\
& =24 n^{2}-40 n-16+32 n+4+\left[16 n^{3}-9 n^{2}-55 n+18\right]-12 n \\
& =16 n^{3}+15 n^{2}-75 n+6
\end{aligned}
$$

$$
\begin{aligned}
C^{\xi}(P(n, n)) & =\sum_{v \in V(P(n, n))} d_{v} / \varepsilon(v) \\
& =2\left(\frac{2}{4 n-1}\right)+4\left(\frac{2}{4 n-2}\right)+4\left(\frac{2}{4 n-4}\right)+4\left(\frac{2}{4 n-6}\right)+\ldots+4\left(\frac{2}{2 n+4}\right)+4\left(\frac{2}{2 n+2}\right)+4\left(\frac{2}{2 n+1}\right) \\
& +2(n-1)\left(\frac{3}{2 n}\right)+2 n\left(\frac{3}{2 n+1}\right)+2(n-1)\left(\frac{3}{2 n+2}\right)+2(n-1)\left(\frac{3}{2 n+3}\right)+2(n-2)\left(\frac{3}{2 n+4}\right) \\
& +2(n-2)\left(\frac{3}{2 n+5}\right)+\ldots+6\left(\frac{3}{4 n-6}\right)+6\left(\frac{3}{4 n-5}\right)+4\left(\frac{3}{4 n-4}\right)+4\left(\frac{3}{4 n-3}\right) \\
& =\frac{4}{4 n-1}+\sum_{i=0}^{n-2}\left(\frac{8}{2 n+2 i+2}\right)+\frac{8}{2 n+1}+\sum_{i=0}^{n-2} 2(n-i)\left(\frac{3}{2 n+2 i}+\frac{3}{2 n+2 i+1}\right)-\left(\frac{6}{2 n}\right) \\
& =\frac{4}{4 n-1}+\sum_{i=0}^{n-2}\left(\frac{8}{2 n+2+2 i}\right)+\frac{8}{2 n+1}+6 \sum_{i=0}^{n-2}\left(\frac{n-i}{4 n^{2}+2 n+4 i^{2}+2(4 n+1) i}\right)-\left(\frac{3}{n}\right)
\end{aligned}
$$




$$
=3 \sum_{i=0}^{n-2}\left(\frac{n-i}{2 n^{2}+n+2 i^{2}+(4 n+1) i}\right)+\sum_{i=0}^{n-2}\left(\frac{4}{n+i+1}\right)+\left(\frac{16 n^{2}-10 n+3}{n\left(8 n^{2}+2 n-1\right)}\right)
$$

Thus $\forall n \geq 1$, the connective eccentric index of $P(n, n)$ is equal to

$$
C^{\xi}(P(n, n))=\sum_{i=0}^{n-2}\left(\frac{5 i^{2}+(16 n+1) i+(11 n+7) n}{2 i^{3}+3(2 n+1) i^{2}+\left(6 n^{2}+6 n+1\right) i+\left(2 n^{2}+3 n+1\right) n}\right)+\left(\frac{16 n^{2}-10 n+3}{n\left(8 n^{2}+2 n-1\right)}\right)
$$

and this completed the proof of Theorem 1.

\section{CONCLUSION}

In this paper, we compute some results of Connective Eccentric index for a connected graph $G$ that defined as $C^{\xi}(G)=\sum_{v \in(G)} \frac{d_{v}}{\varepsilon(v)}$. In finally, we introduce a closed formula of $C^{\xi}$ for a molecular graph " linear polycene parallalogram benzenoid graph $P(n, n) "$

\section{Acknowledgments}

The author is thankful to Professor Dr. Mehdi Alaeiyan from Department of Mathematics, Iran University of Science and Technology (IUST), and Professor Mircea V. Diudea from the Faculty of Chemistry and Chemical Engineering of Babes-Bolyai University (Romania) for their precious support and suggestions.

\section{References}

[1] H. Wiener, J. Am. Chem. Soc. 69(1) (1947) 17-20.

[2] I. Gutman, N. Trinajstić, Chem. Phys. Lett. 17 (1972) 535-538.

[3] R.Todeschini, V. Consonni, Handbook of Molecular Descriptors, Weinheim, Wiley$\mathrm{VCH},(2000)$.

[4] V. Sharma, R. Goswami, A. K. Madan, J. Chem. Inf. Comput. Sci. 37 (1997) 273-282.

[5] S. Alikhani, M. A. Iranmanesh. Digest. J. Nanomater. Bios. 6(1) (2011) 253-257.

[6] A. R. Ashrafi, M. Ghorbani, M. Hemmasi. Digest. J. Nanomater. Bios. 4(3) (2009) 483-486.

[7] A. R. Ashrafi, M. Ghorbani, M. Jalali, Optoelectron. Adv. Mater.-Rapid Comm. 3 (2009) 823-826.

[8] H. Dureja, A. K. Madan, Med. Chem. Res. 16 (2007) 331-341.

[9] M. Fischermann, A. Homann, D. Rautenbach, L. A. Szekely, L. Volkmann, Discrete Appl. Math. 122 (2002) 127-137.

[10] M. Ghorbani, M. Ghazi, Digest. J. Nanomater. Bios. 5(4) (2010) 1107-1111. 
[11] I. Gutman, O. E. Polansky, Mathematical Concepts in Organic Chemistry, SpringerVerlag, New York, (1986). M.A. Johnson, G.M. Maggiora. Concepts and Applications of Molecular Similarity, Wiley Interscience, New York, (1990).

[12] V. Kumar, S. Sardana, A. K. Madan, J. Mol. Model 10 (2004) 399-407.

[13] S. Sardana, A. K. Madan, MATCH Commun. Math. Comput. Chem. 43 (2001) 85-89.

[14] Z. Yar Ahmadi, Iran. J. Math. Chem. 1(2) (2010) 105-110.

[15] M. Alaeiyan, R. Mojarad, J. Asadpour, Optoelectron. Adv. Mater.-Rapid Commun. 5(7) (2011) 761-763.

[16] S. Gupta, M. Singh, A. K. Madan, J. Mol. Graph. Model. 18 (2000) 18-25.

[17] S. Gupta, M. Singh, A. K. Madan, J. Math. Anal. Appl. 266 (2002) 259-268.

[18] P. V. Khadikar, Iranian Journal of Mathematical Chemistry 1(1) (2010) 7-42.

[19] M. Alaeiyan, J. Asadpour, Optoelectron. Adv. Mater.-Rapid Commun. 6(1-2) (2012) 191-193.

[20] Mohammad Reza Farahani, International Letters of Chemistry, Physics and Astronomy 11(1) (2014) 74-80.

[21] Mohammad Reza Farahani, International Letters of Chemistry, Physics and Astronomy 12 (2014) 56-62.

[22] Mohammad Reza Farahani, International Letters of Chemistry, Physics and Astronomy 12 (2014) 63-68.

[23] Mohammad Reza Farahani, International Letters of Chemistry, Physics and Astronomy 13(1) (2014) 1-10.

[24] Mohammad Reza Farahani, International Letters of Chemistry, Physics and Astronomy 17(2) (2014) 201-206. 